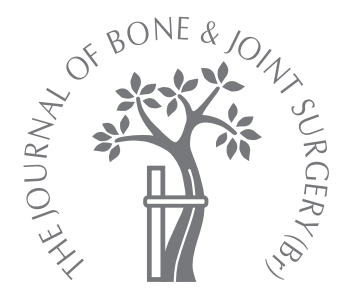

\title{
Embolic phenomena during computer- assisted and conventional total knee replacement
}

\author{
J. S. Church, \\ J. E. Scadden, \\ R. R. Gupta, \\ C. Cokis, \\ K. A. Williams, \\ G. C. Janes
}

From Perth

Orthopaedic and

Sports Medicine

Centre, Perth,

Western Australia

J. S. Church, BSc, FRCS(Orth), Locum Consultant Orthopaedic Surgeon

Department of Orthopaedics

Chelsea \& Westminster Hospital, 369 Fulham Road, London SW10 $9 N H$, UK.

- J. E. Scadden, FRCS(Orth), Consultant Orthopaedic Surgeon St. Mary's Hospital, Newport, Isle of Wight PO30 5TG, UK.

R. R. Gupta, FRCS(Orth), Consultant Orthopaedic Surgeon St. Peter's Hospital, Chertsey, Surrey KT16 OPZ, UK.

C. Cokis, FANZCA, Consultant Anaesthetist

Hollywood Hospital, Monash Avenue, Perth 6009, Western Australia.

K. A. Williams, FANZCA Consultant Anaesthetist Mount Hospital, 146 Mounts Bay Road, Perth 6000, Western Australia.

G. C. Janes, FRACS(Orth), Consultant Orthopaedic Surgeon Perth Orthopaedic \& Sports Medicine Centre, 31 Outram Street, West Perth 6005, Western Australia.

Correspondence should be sent to Mr J. S. Church; e-mail: j.s.church@btinternet.com

C2007 British Editorial Society of Bone and Joint Surgery doi:10.1302/0301-620X.89B4. $18470 \$ 2.00$

$J$ Bone Joint Surg $[\mathrm{Br}]$ 2007:89-B:481-5.

Received 31 July 2006,

Accepted after revision

19 December 2006

\begin{abstract}
Systemic emboli released during total knee replacement have been implicated as a cause of peri-operative morbidity and neurological dysfunction. We undertook a prospective, double-blind, randomised study to compare the cardiac embolic load sustained during computer-assisted and conventional, intramedullary-aligned, total knee replacement, as measured by transoesophageal echocardiography. There were 26 consecutive procedures performed by a single surgeon at a single hospital. The embolic load was scored using the modified Mayo grading system for echogenic emboli.

Fourteen patients undergoing computer-assisted total knee replacement had a mean embolic score of 4.89 ( 3 to 7 ) and 12 undergoing conventional total knee replacement had a mean embolic score of 6.15 (4 to 8 ) on release of the tourniquet. Comparison of the groups using a two-tailed $t$-test confirmed a highly significant difference $(p=0.004)$.

This study demonstrates that computer-assisted knee replacement results in the release of significantly fewer systemic emboli than the conventional procedure using intramedullary alignment.
\end{abstract}

Systemic embolic phenomena are well recognised during total knee replacement (TKR). ${ }^{1-6}$ They are widely believed to be the cause of intra-operative hypotension and reduced cardiac output, which may lead to circulatory collapse and sudden death. ${ }^{7-9}$ Emboli have been variously described as consisting of elements of marrow, ${ }^{2,3}$ thrombus, ${ }^{1}$ fat $^{2,10}$ and bone particles ${ }^{11}$ released during preparation of the femur and tibia.

The advent of transoesophageal echocardiography (ECG) has enabled the monitoring and quantification of these emboli, and some studies have confirmed that techniques of extramedullary alignment result in a reduced cardiac embolic load compared with intramedullary methods. ${ }^{3,5}$ Other studies, however, have found no difference ${ }^{4,6}$ and suggest that the emboli are composed of venous thrombus caused by tourniquet-induced venous stasis. There have also been concerns about the accuracy of existing methods of extramedullary alignment, ${ }^{12,13}$ as a result of which most surgeons continue to use intramedullary alignment rods when performing TKR.

Computer-assisted navigation in TKR is becoming more popular and has several potential advantages over conventional procedures. There is considerable evidence in the literature that computer navigation results in greater accuracy and reproducibility by way of improved alignment and control of rotation. ${ }^{14-16}$ There is also evidence of a significant reduction in peri-operative blood loss compared with conventional techniques. ${ }^{16,17}$ Computerassisted TKR is performed without the use of intramedullary alignment rods and potentially may result in fewer embolic complications. This prospective study was designed to compare the cardiac embolic load during both computer-assisted and conventional TKR.

\section{Patients and Methods}

After approval by the local ethics committee, patients awaiting primary TKR for osteoarthritis were invited to take part in the study. Exclusion criteria included a history of inflammatory arthritis, previous femoral instrumentation, thromboembolic disease and an oesophageal disorder. Informed consent was obtained from 26 consecutive patients who met the criteria and who were subsequently blinded as to the method of their knee replacement.

Immediately before surgery the patients were randomised to undergo either conventional TKR using intramedullary alignment or computer-assisted TKR. Randomisation was achieved using a computerised random number generator (Microsoft Excel, Red- 


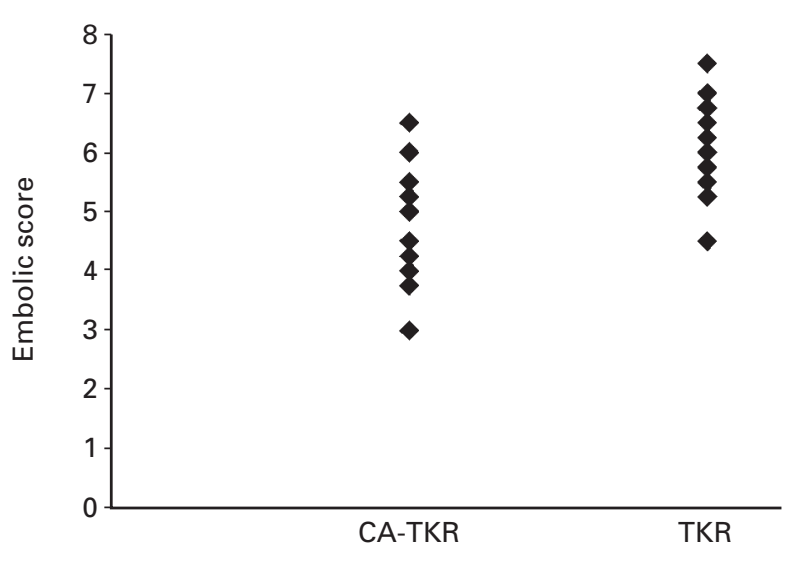

Fig. 1

Comparison of mean embolic scores in patients undergoing computerassisted total knee replacement (CA-TKR) against those undergoing conventional total knee replacement (TKR).

mond, Washington). All procedures were performed with the use of a tourniquet, applied to the thigh to a pressure of $350 \mathrm{mmHg}$. The tourniquet was inflated prior to antiseptic preparation of the skin and deflated after closure and dressing of the incision in all cases. The patients did not receive peri-operative thromboprophylaxis.

All patients were given a general anaesthetic and underwent endotracheal intubation before the insertion of a $5 \mathrm{mHz}$ multiplane transoesophageal ECG probe (Sequoia Echocardiography Platform C512, Siemens, Munich, Germany). Continuous recordings from the right atrium and ventricle began just prior to inflation of the tourniquet and continued until five minutes after the tourniquet was deflated. The exact times of tourniquet inflation, femoral breach, tibial breach, first cementation and tourniquet release were recorded to assist in identifying the cause of any embolic 'showers' which were seen.

All operations were carried out by the senior author (GCJ). All patients received the same cruciate-retaining prosthesis (distal cut Genesis II, Smith \& Nephew, Memphis, Tennessee) and the patellae were not resurfaced. Intramedullary femoral and extramedullary tibial alignment was used in the conventional TKR group. The fluted intramedullary rod was inserted through an enlarged (9.5 $\mathrm{mm}$ ) femoral entry hole, in accordance with the manufacturer's instructions. In the computer-assisted group, femoral and tibial alignment was achieved using the VectorVision navigation system (BrainLAB, Heimstetten, Germany). This required the percutaneous insertion of two $3.2 \mathrm{~mm}$ threaded pins across the tibial and femoral canals, on which to secure the reflective sphere arrays. No further intramedullary instrumentation was necessary.

Two anaesthetists (CC, KAW), with over 20 years' combined experience in intra-operative transoesophageal ECG, independently reviewed the videotapes of the ECG images obtained during each operation. The observers were blinded as to whether the patient had undergone conventional or computer-assisted surgery. They were asked to score each embolic event using the modified Mayo Clinic grading system for echogenic emboli, ${ }^{1,18}$ which gives a total score of three to nine points depending on the percentage of the atrium filled, the duration of echogenesis and the diameter of the largest particles (Table I). Each observer was asked to review the videotapes a second time approximately six weeks later.

Before the study it was not known exactly when embolic showers would be seen and what scores each shower would achieve. It quickly became apparent that, although a few small showers were seen intra-operatively, these were insignificant compared with that seen upon release of the tourniquet. The small showers were not related to the type of procedure performed and did not occur at any consistent point during the operation. We did not observe an increase in embolic phenomena in any patient at the time of insertion of the intramedullary femoral rod. The decision was therefore taken to focus our statistical analysis on the scores obtained when the tourniquet was released.

The mean of the four 'tourniquet' scores obtained for each operation was calculated and the data produced were statistically analysed using the two-tailed $t$-test. Intra- and inter-observer variabilities were assessed using two-way ANOVA and the paired $t$-test, respectively.

\section{Results}

Complete data were recorded for all 26 patients. There were 14 patients (ten male, four female) in the computerassisted TKR group and 12 (four male, eight female) in the conventional TKR group. There was no significant difference between the embolic scores of males or females in either group. The groups did not differ significantly in age, weight or body mass index (Table II) and there was no correlation between any of these parameters and the embolic score. Operation time was significantly longer in the computer-assisted group by a mean of 17 minutes (mean operative time: computer-assisted group 74.1 minutes (60 to 98$)$, conventional group 56.8 minutes (49 to 63$)$ ) $(\mathrm{p}=0.0003)$.

Patients undergoing conventional TKR had a mean embolic score of 6.15 (4 to 8) on release of the tourniquet. Those undergoing computer-assisted TKR had a mean embolic score of 4.89 ( 3 to 7). Comparison of the groups using a twotailed $t$-test shows a significant difference ( $\mathrm{p}=0.004$; Fig. 1$)$.

Intra-observer replication had a variance of 0.2 , giving a $95 \%$ confidence limit of 0.9 units for a single observation. Analysis of inter-observer variability revealed a significant difference of 0.48 units between our two scores ( $p=0.001$ ). In $24(92 \%)$ of the cases, the mean scores produced by the scorers were within one unit of each other.

No emboli larger than $0.5 \mathrm{~cm}$ were seen during any of the operations. There were no significant intra- or post-operative complications, including wound infection (superficial or deep), chest infection, pulmonary embolism or deep venous thrombosis. 
Table I. The modified Mayo Clinic grading system for echogenic emboli ${ }^{1}$

\begin{tabular}{llll}
\hline Score & \% filling of right atrium & Duration of echogenesis (seconds) & Diameter of largest particle (cm) \\
\hline 1 & $<50$ & $<25$ & $<0.5$ \\
2 & 50 to 75 & 25 to 35 & 0.5 to 1.0 \\
3 & $>75$ & $>35$ & $>1.0$ \\
\hline
\end{tabular}

Table II. Mean (range) differences between the two groups and their significance

\begin{tabular}{|c|c|c|c|}
\hline & CA-TKR ${ }^{*}$ & $\mathrm{TKR}^{\dagger}$ & p-value \\
\hline Age in yrs & $62.3 \quad(52$ to 75$)$ & 67.1 (54 to 80$)$ & $0.18(\mathrm{NS})^{\ddagger}$ \\
\hline Weight in kg & $91.8 \quad$ (68 to 116$)$ & $82.2(66$ to 110$)$ & 0.10 (NS) \\
\hline Body mass index in $\mathrm{kg} / \mathrm{m}^{2}$ & $31.6 \quad$ (23.5 to 40.2 ) & $29.6 \quad(23.4$ to 43.0$)$ & 0.37 (NS) \\
\hline Operation time in minutes & $74.1 \quad$ (60 to 98$)$ & 56.8 (49 to 63$)$ & 0.0003 \\
\hline Embolic score & 4.89 (3 to 7 ) & 6.15 (4 to 8 ) & 0.004 \\
\hline
\end{tabular}

* CA-TKR, computer-assisted total knee replacement

† TKR, total knee replacement

$\ddagger$ NS, not significant

\section{Discussion}

This study demonstrates that significantly fewer echogenic emboli are released into the cardiovascular system during computer-assisted TKR than in conventional TKR. This supports the recent study by Kalairajah et $\mathrm{al}^{19}{ }^{19}$ who observed reduced cerebral emboli detected by transcranial Doppler ultrasonography in computer-assisted TKR. Their use of a non-invasive investigation only enabled the detection of emboli that had passed through the pulmonary vasculature or bypassed it via a right-to-left shunt (e.g. patent foramen ovale). The use of the more invasive transoesophageal ECG probe in our study enabled us to confirm that the true systemic embolic load is also significantly reduced.

The insertion of a femoral intramedullary guide was the only invasive procedure performed during the conventional TKR that was not undertaken during the computer-assisted operations. It is therefore reasonable to assume that this was the cause of the increased embolic load. This assumption is supported by the findings of Morawa et al, ${ }^{5}$ who observed significantly fewer emboli when using an extramedullary femoral alignment guide. They found a similar but less significant effect when comparing extramedullary and intramedullary techniques of tibial alignment. Markel et $\mathrm{al}^{3}$ similarly concluded that intramedullary guides increase the generation of debris in dogs.

Takahashi, Kitagawa and Ishii ${ }^{20}$ observed moderate to severe embolic events in $80 \%$ of patients undergoing instrumented spinal surgery but none in non-instrumented patients, and Duwelius et $\mathrm{al}^{10}{ }^{10}$ when performing intramedullary nailing on sheep femora, found that the operative manoeuvre associated with the greatest embolic release was opening the intramedullary canal with an awl. This evidence would appear to support the theory that embolic release is directly proportional to the degree of violation of the femoral canal.
In 1995, however, Parmet et $\mathrm{al}^{6}$ found that there was no significant difference in the number of large venous emboli seen when performing intramedullary- and extramedullaryaligned TKR. In a later study, ${ }^{4}$ they stated again that marrow cavity invasion is not correlated to the release of large emboli, but that use of a tourniquet increases the risk fivefold. They were the first to suggest that there were different types of embolus. ${ }^{21}$ Small 'miliary' emboli, presumably consisting of elements of marrow, are seen in all TKRs, but may be more likely to occur with intramedullary instrumentation. They suggest that large emboli occur regardless of femoral instrumentation. These are responsible for producing the intense physiological response which may lead to circulatory collapse, and appear to be related to the use of a tourniquet. They suggested that these emboli consist of venous thrombus caused by tourniquet-induced stasis. ${ }^{6}$ A further study by Berman et al $^{1}$ at the same institution found that the pulmonary vascular resistance index increased after release of the tourniquet only in patients who had echogenic material of at least $0.5 \mathrm{~cm}$ in diameter. Blood aspirated from pulmonary artery catheters in these patients was found to contain fresh venous thrombus. It is worth noting that no emboli larger than $0.5 \mathrm{~cm}$ in diameter were seen in our study, despite the use of a tourniquet.

The clinical relevance of our study is based on the premise that there is a relationship between echogenic emboli and peri-operative morbidity. In 1997, Lafont et $\mathrm{al}^{22}$ found no correlation between frequency or size of embolic particles and blood gas and haemodynamic variables (heart rate, arterial blood pressure and central venous pressure) during total hip replacement. The majority of published articles disagree with this finding, however. Christie et $\mathrm{al}^{7}$ found that pulmonary responses correlated with the severity of embolic phenomena during invasive intramedullary procedures in humans, and Wheelwright et $\mathrm{al}^{8}$ observed that hypotension and decreased cardiac output were directly 
related to the magnitude of pulmonary embolism in dogs undergoing bilateral cemented arthroplasty.

$\mathrm{Kim}^{2}$ took blood from the right atrium of patients undergoing TKR and found a significant increase in the number of fat emboli 5 to 10 minutes after insertion of the femoral alignment rod. In $12 \%$ of patients undergoing bilateral TKR and $4 \%$ of those undergoing unilateral TKR there were also bone marrow cells in the blood samples. These patients had significantly lower arterial oxygen tension and oxygen saturation on the first post-operative day than did those without marrow cells in the samples. This not only provides direct evidence of a measurable clinical response resulting from embolism, but also suggests that embolic content is as important as size.

$\mathrm{Kim}^{2}$ also showed that patients undergoing bilateral TKR have a significantly increased risk of fat and marrow embolism compared with those undergoing unilateral TKR. This finding is supported by Dorr et al, ${ }^{23}$ who quote a $12 \%$ incidence of fat embolism during bilateral TKR which most often manifests as a change of mental status. They suggest that a sustained fall in oxygen saturations below $90 \%$ would be an indication to abort the second knee arthroplasty. Bullock, Sporer and Shirreffs ${ }^{24}$ and Oakes and Hanssen ${ }^{25}$ also found an increase in cardiovascular and neurological complications following bilateral rather than unilateral TKR. Our finding of a significant reduction in systemic embolic load with the use of computer-assisted TKR would appear to support its use in these potentially high-risk surgical procedures. Similar conclusions could be applied to patients with a patent foramen ovale, which may be as many as $25 \%$ of the population according to post-mortem studies. ${ }^{26}$

One potential weakness of this study is the quantification of emboli with real-time ultrasound. We have used previously published techniques and methods of assessment, but the true embolic load cannot be accurately quantified with any currently known technique. However, scoring of embolic showers using real-time ultrasound remains the best means of comparative assessment of these phenomena. It is our opinion that the scores obtained are at least proportional to the embolic insult.

Another potential weakness of this study, and all studies quantifying intra-operative cardiac embolic phenomena, is that there are no data on what constitutes a 'safe' embolic load. Although we are able to prove a statistically significant observed decrease in embolic phenomena when using computer-assisted TKR, we cannot prove that this equates to a clinically significant decrease. It is likely that there is considerable inter-individual variation in the embolic threshold, beyond which pathophysiological cardiopulmonary responses develop. It is also likely that this threshold is reduced in the significant proportion of elderly patients undergoing TKR who have reduced cardiopulmonary reserve. Given that $\mathrm{Kim}^{2}$ has demonstrated that embolic insult can result in measurable pathophysiological responses, we feel that the responsible surgeon should pur- sue all means to reduce this, provided the surgical outcome is not compromised.

Analysis of the scoring system itself has revealed minimal intra-observer variability. Although there was a statistically significant inter-observer difference, this equated to fewer than 0.5 units per patient. Our figure of $92 \%$ agreement or disagreement within one unit between observers exactly matches the figure of Ereth et $a{ }^{18}{ }^{18}$ who developed the scoring system.

A further concern with this study is the relatively few patients in each group. A visible difference in the quantity of emboli and the duration of the showers was obvious soon after commencement of the study, however, and statistical significance was reached after ten patients. A further 16 patients were entered into the study to reduce the risk of a type I error but, in view of the increasingly significant difference the study was abandoned and the senior author (GCJ) reverted to performing solely computer-assisted TKR.

As one might expect, this study demonstrated a moderate increase in the operative time when performing computerassisted TKR, as reported in previous studies. ${ }^{16,27}$ We did not encounter any problems associated with this, however, and it is our opinion that this increase is more than compensated for by the improved accuracy, reproducibility and margin of safety provided by this particular surgical technique.

This study demonstrates that computer-assisted TKR results in significantly fewer systemic embolic events than conventional TKR using intramedullary alignment. This is likely to reduce the risk of peri-operative cardiovascular complications and post-operative neurological dysfunction. In the light of recent studies confirming improved accuracy and reproducibility, as well as reduced blood loss, this would appear to add to the growing list of compelling arguments in favour of computer-assisted TKR.

We wish to thank Echo Services (Hollywood Specialist Centre, Nedlands, Perth, Western Australia) for providing the transoesophageal echocardiographic equipment and Mr Livio Mina for his assistance with the statistical analysis.

No benefits in any form have been received or will be received from a commercial party related directly or indirectly to the subject of this article.

\section{References}

1. Berman AT, Parmet JL, Harding SP, et al. Emboli observed with use of transoesophageal echocardiography immediately after tourniquet release during total knee arthroplasty with cement. J Bone Joint Surg [Am] 1998;80-A:389-96.

2. Kim YH. Incidence of fat embolism syndrome after cemented or cementless bilateral simultaneous and unilateral total knee arthroplasty. J Arthroplasty 2001;16:730-9.

3. Markel DC, Femino JE, Farkas P, Markel SF. Analysis of lower extremity embolic material after total knee arthroplasty in a canine model. J Arthroplasty 1999;14:22732.

4. Parmet JL, Horrow JC, Berman AT, et al. The incidence of large venous emboli during total knee arthroplasty without pneumatic tourniquet use. Anesth Analg 1998;87:439-44

5. Morawa LG, Manley MT, Edinin AA, Reilly DT. Transoesophageal echocardiographic monitored events during total knee arthroplasty. Clin Orthop 1996;331:192-8.

6. Parmet JL, Horrow JC, Pharo G, et al. The incidence of venous emboli during extramedullary guided total knee arthroplasty. Anesth Analg 1995;81:757-62.

7. Christie J, Robinson CM, Pell AC, McBirnie J, Burnett R. Transcardiac echocardiography during invasive intramedullary procedures. J Bone Joint Surg $[B r] 1995 ; 77-$ B: $450-5$. 
8. Wheelwright EF, Byrick RJ, Wigglesworth DF, et al. Hypotension during cemented arthroplasty: relationship to cardiac output and fat embolism. J Bone Joint Surg $[B r] 1993 ; 75-B: 715-23$.

9. Monto RR, Garcia J, Callaghan JJ. Fatal fat embolism following total condylar knee arthroplasty. J Arthroplasty 1990;5:291-9.

10. Duwelius PJ, Huckfeldt R, Mullins RJ, et al. The effects of femoral intramedullary reaming on pulmonary function in a sheep lung model. J Bone Joint Surg [Am] 1997;79-A:194-202

11. Hayakawa M, Fujioka Y, Morimoto Y, Okamura A, Kemmotsu O. Pathological evaluation of venous emboli during total hip arthroplasty. Anaesthesia 2001;56:5715.

12. Reed MR, Bliss W, Sher JL, et al. Extramedullary or intramedullary tibial alignment guides: a randomised, prospective trial of radiological alignment. J Bone Joint Surg [Br] 2002;84-B:858-60.

13. Maestro A, Harwin SF, Sandoval MG, Vaquero DH, Murcia A. Influence of intramedullary versis extramedullary alignment guides on final total knee arthroplasty component position: a radiographic analysis. J Arthroplasty 1998;13:552-8.

14. Anderson KC, Buehler KC, Markel DC. Computer assisted navigation in total knee arthroplasty: comparison with conventional methods. J Arthroplasty 2005:20:132-8.

15. Bathis $\mathbf{H}$, Perlick L, Tingart $\mathbf{M}$, et al. Alignment in total knee arthroplasty: a comparison of computer-assisted surgery with the conventional technique. J Bone Joint Surg [Br] 2004;86-B:682-7.

16. Chauhan SK, Scott RG, BreidahI W, Beaver RJ. Computer-assisted knee arthroplasty versus a conventional jig-based technique: a randomised, prospective trial. $J$ Bone Joint Surg [Br] 2004;86-B:372-7.

17. Kalarajah Y, Simpson D, Cossey AJ, Verrall GM, Spriggins AJ. Blood loss after total knee arthroplasty: effects of computer-assisted surgery. J Bone Joint Surg [Br] 2005;87-B:1480-2
18. Ereth MH, Weber JG, Abel MD, et al. Cemented versus noncemented total hip arthroplasty: embolism, hemodynamics and intrapulmonary shunting. Mayo Clin Proc 1992:67:1066-74.

19. Kalairajah Y, Cossey AJ, Verrall GM, Ludbrook G, Spriggins AJ. Are systemic emboli reduced in computer-assisted knee surgery: a prospective, randomised, classical trial. J Bone Joint Surg [Br]2006;88-B:198-202.

20. Takahashi S, Kitagawa H, Ishii T. Intraoperative pulmonary embolism during spinal instrumentation surgery: a prospective study using transoesophageal echocardiography. $J$ Bone Joint Surg [Br] 2003;85-B:90-4

21. Parmet JL, Horrow JC, Singer R, Berman AT, Rosenberg H. Echogenic emboli upon tourniquet release during total knee arthroplasty: pulmonary hemodynamic changes and embolic composition. Anesth Analg 1994;79:940-5.

22. Lafont ND, Lakonji MK, Barre J, Guillaume C, Booggaerts JG. Clinical feature and echocardiography of embolism during cemented hip arthroplasty. Can J Anaesth 1997:44:112-17.

23. Dorr LD, Merkel C, Mellman MF, Klein I. Fat emboli in bilateral total knee arthroplasty: predictive factors for neurologic manifestations. Clin Orthop 1989:248:112-18.

24. Bullock DP, Sporer SM, Shirreffs TG Jr. Comparison of simultaneous bilateral with unilateral total knee arthroplasty in terms of perioperative complications. J Bone Joint Surg [Am]2003;85-A:1981-6.

25. Oakes DA, Hanssen AD. Bilateral total knee replacement using the same anesthetic is not justified by assessment of the risks. Clin Orthop 2004;428:87-91.

26. Hagen PT, Scholz DG, Edwards WD. Incidence and size of patent foramen ovale during the first 10 decades of life: an autopsy study of 965 normal hearts. Mayo Clin Proc 1984:59:17-20

27. Bolognesi M, Hofmann A. Computer navigation versus standard instrumentation for TKA: a single-surgeon experience. Clin Orthop 2005;440:162-9. 